\title{
ÚNG DỤNG TIN HỌC VÀO CÁC BÀI TOÁN TRĂC ĐỊA TRONG KHẢO SÁT, THIẾT KẾ VÀ THI CÔNG XÂY DỰNG ĐƯờNG HẦM
}

\author{
TS. ĐINH CÔNG HOÀ(1), ThS. HOÀNG TH! THỦY(1), KS. NGUYẼN VĂN LÂM(2) \\ (1)Trường Đại học Mỏ - Địa chất \\ ${ }^{(2)}$ Công ty Khai thác Khoảng sản Tây Nguyên, Tổng Công ty Đông Bắc, Bộ Quốc phòng
}

\section{Mở đầu}

Những năm gần đây, công nghệ và thiết bị hiện đại không ngừng phát triển, đang được ứng dụng rộng rãi trong công tác trắc địa. Đối với cơ sở dữ liệu địa hình, bản đồ được thành lập trong các hệ quy chiếu khác nhau (HN72, VN2000) và với các kinh tuyến trục khác nhau. Dữ liệu bản đồ số địa hình của các đơn vị sản xuất cũng rất đa dạng, được thành lập bằng các phần mềm khác nhau. Vì vậy nhu cầu của thực tế sản xuất cần chuyển đổi bản đồ về cùng hệ toạ độ thống nhất để thuận lợi cho công tác quản lý và sử dụng, đặc biệt là trong công tác khảo sát thiết kế và thi công đường hầm. Trong công tác thi công xây dựng đường hầm, việc định tuyến hầm theo các phương nằm ngang và thẳng đứng, độ cong ngang, cong đứng, độ dốc và mặt cắt hầm ở các vị trí khác nhau, đòi hỏi phải xác định chính xác, kịp thời và kết quả xử lý cần trực quan thông qua số liệu thiết kế và đo đạc.

Phần mềm Geosoft đã có các chức năng tự động chuyển đổi bản đồ về hệ toạ độ mong muốn từ dữ liệu ở các hệ quy chiếu và khuôn dạng khác nhau, đảm bảo bảo toàn mối quan hệ hình học của các đối tượng không gian và không làm thay đổi các thuộc tính của đối tượng. Trên cơ sở số liệu thiết kế và dữ liệu đo đạc, phần mềm Geosoft có thể tự động hóa xác định hướng đường hầm theo phương nằm ngang và thẳng đứng, tính khối lượng đào trong thi công xây dựng hầm. Các kết quả tính toán chính xác, trực quan thể hiện trên phần mềm đồ họa AutoCad.

\section{2. Ứng dụng tin học trong thi công xây dựng đường hầm}

Trong quá trình thi công đường hầm, căn cứ số liệu đo vẽ hoàn công, chúng ta tiến hành đo và vẽ mặt cắt ngang hầm, tính diện tích thiết diện đường hầm và từ đó so sánh với giá trị thiết kế, xác định khoảng lệch giữa thi công và thiết kế để kịp thời điều chỉnh trong quá trình thi công, cũng như công tác đo vẽ hoàn công công trình. Để có giá trị thiết kế mặt cắt ngang hầm chúng ta phải thiết lập tệp tin về tuyến đường hầm. Tệp tin này là cơ sở cho tất cả công tác thành lập mặt cắt ngang hầm đối với một công trình. Cấu trúc tệp tin gồm tọa độ $\mathrm{x}, \mathrm{y}$, h, độ dốc, bán kính cong ngang, bán kính cong đứng các điểm ngoặt tim hầm. Tương ứng với các điểm này là thông tin về mặt cắt thiết kế hầm (xem hình 1, 2, 3).
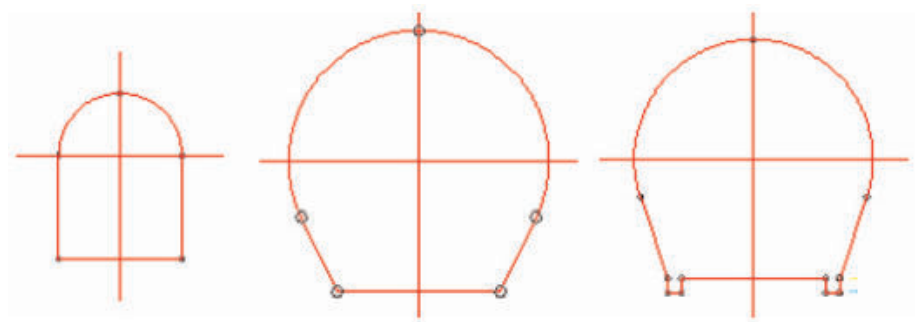

Hình 1: Mặt cắt thiết kế hầm 


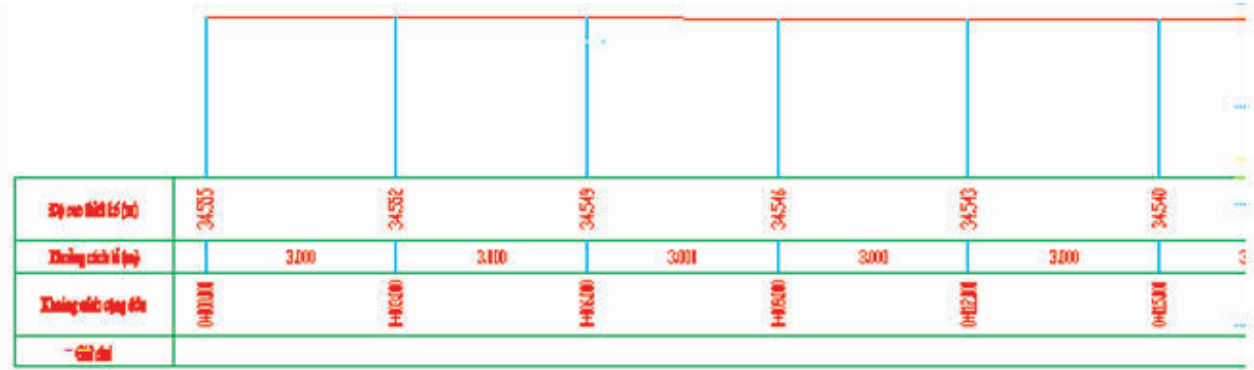

Hình 2: Mặt cắt dọc tim hầm toàn tuyến

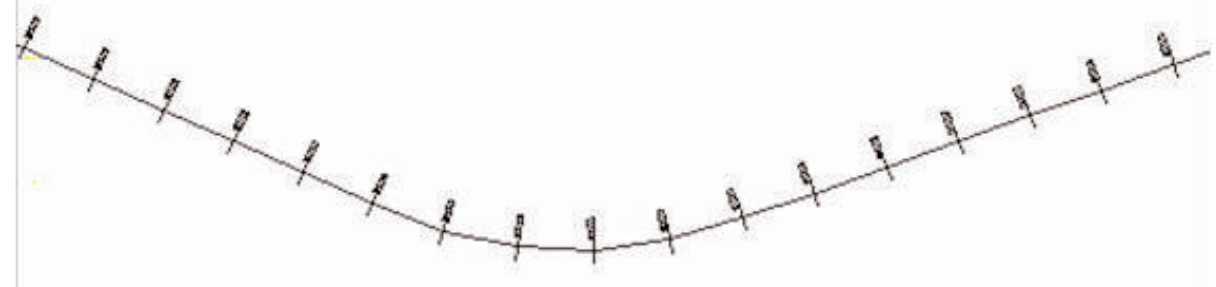

Hình 3: Bình đồ vị trí mặt bằng các mặt cắt hầm toàn tuyến

Trên cơ sở các thông tin trên, chúng ta xác định được vị trí tim hầm, mặt cắt thiết kế và mặt cắt thi công tại mọi vị trí của hầm.

Mặt cắt ngang hầm được vẽ đúng kích thước, một đường theo thiết kế, một đường theo số liệu đo hoàn công. Tại các điểm đo hoàn công xác định giá trị lệch so với thiết kế theo phương gần nhất. Giá trị lệch được hiển thị ngay trên bản vẽ, kể cả giá trị lệch của điểm đo theo chiều dọc tuyến.

\section{Chuyển đổi bản đồ số địa hình bằng phần mềm ứng dụng Geosoft}

a) Công thức tính chuyển từ tọa độ trắc địa $(B, L)$ sang tọa độ vuông góc phẳng $(x, y)$

$$
\begin{gathered}
(x-X)=\frac{N}{2 \rho^{\prime \prime 2}} \sin B \cos B 1^{\prime 2}+\frac{N}{24 \rho^{\prime \prime}} \sin B \cos ^{3} B\left(5-t^{2}+9 \eta^{2}+4 \eta^{2}\right) 1^{14}+ \\
+\frac{N}{720 \rho^{\prime \prime 6}} \sin B \cos ^{5} B\left(61-58 t^{2}+t^{4}\right) 1^{\prime \prime} \\
y=\frac{N}{\rho^{\prime \prime}} \cos B 1^{\prime \prime}+\frac{N}{6 \rho^{\prime \prime 3}} \cos ^{3} B\left(1-t^{2}+\eta^{2}\right) 1^{\prime \prime}+\frac{N}{120 \rho^{\prime \prime}} \cos ^{5} B\left(5-18 t^{2}+t^{4}+14 \eta^{2}-58 t^{2} \eta^{2}\right) 1^{11^{5}}
\end{gathered}
$$

b) Công thức tính chuyển từ tọa độ vuông góc phẳng $(x, y)$ sang tọa độ trắc địa $(B, L)$

$$
\begin{aligned}
B_{1}-B & =\frac{\rho^{\prime \prime} t_{1}}{2 M_{1} N_{1}} y^{2}-\frac{\rho^{\prime \prime} t_{1}}{2 M_{1} N_{1}^{3}}\left(5+3 t_{1}^{2}+\eta_{1}^{2}-9 t_{1}^{2} \eta_{1}^{2}\right) y^{4}+ \\
& +\frac{\rho^{\prime \prime} t_{1}}{720 M_{1} N_{1}^{5}}\left(61+90 t_{1}^{2}+45 t_{1}^{2}\right) y^{6}
\end{aligned}
$$




$$
\begin{aligned}
& l=\frac{\rho^{\prime \prime}}{N_{1} \cos B_{1}} y-\frac{\rho^{\prime \prime}}{6 N_{1}^{3} \cos B_{1}}\left(1+2 t_{1}^{2}+\eta_{1}^{2}\right) y^{3}+ \\
& +\frac{\rho^{\prime \prime}}{120 N_{1}^{5} \cos B_{1}}\left(5+28 t_{1}^{2}+24 t_{1}^{4}+6 \eta_{1}^{2}+8 t_{1}^{2} \eta_{1}^{2}\right) y^{5}
\end{aligned}
$$

Trong các công thức trên, $X$ là chiều dài cung kinh tuyến, được xác định dựa vào độ vĩ $B$ đã biết; $\mathrm{N}$ là bán kính cong vòng thẳng đứng thứ nhất; hệ số $\mathrm{I}, \mathrm{M}, \mathrm{N}, \mathrm{t}, \mathrm{h}$ được tính như sau:

$$
\begin{aligned}
& \mathrm{I}=\mathrm{L}-\mathrm{L}_{\mathrm{O}} \quad\left(\mathrm{L}_{\mathrm{o}} \text { là kinh tuyến trục }\right) ; \\
& \mathrm{t}=\operatorname{tg} \mathrm{B} ; \quad \mathrm{t}_{1}=\operatorname{tgB}_{1} ; \quad \mathrm{h}^{2}=\mathrm{e}^{2} \cos ^{2} \mathrm{~B} ; \\
& M=\frac{a\left(1-e^{2}\right)}{\left(1-e^{2} \sin ^{2} B\right)^{3 / 2}} ; \quad N=\frac{a}{\left(1-e^{2} \sin ^{2} B\right)^{1 / 2}}
\end{aligned}
$$

$\mathrm{N}$ là bán kính cong vòng thẳng đứng thứ nhất;

e là độ lệch tâm thứ nhất, e' là độ lệch tâm thứ hai;

a là bán trục lớn của Elipxoid;

\section{c) Tính chuyển toạ độ phẳng bằng phép biến đổi đa thức}

Trong trường hợp khu vực nhỏ hoặc bản đồ được xây dựng trong hệ tọa độ địa phương, chúng ta dùng phép biến đổi đa thức để chuyển đổi dữ liệu bản đồ số địa hình về hệ tọa độ VN2000 dựa vào tọa độ các điểm song trùng. Các điểm song trùng được phân bố đều trên toàn bộ khu vưc chuyển đổi.[1]

$$
\begin{aligned}
& X=A 1+B 1 * X 1+C 1{ }^{*} Y 1+D 1 * X 1 \wedge 2+E 1 * Y 1 \wedge 2+G 1 * X 1 * Y 1 \\
& Y=A 2+B 2 * X 1+C 2 * Y 1+D 2 * X 1 \wedge 2+E 2 * Y 1 \wedge 2+G 2 * X 1{ }^{*}{ }^{*}{ }^{*} 1
\end{aligned}
$$

d) Chuyển đổi bản đồ số địa hình qua các hệ quy chiếu

Từ tệp dữ liệu đồ hoạ của bản đồ số, bằng việc phân tích khuôn dạng dữ liệu [1], chúng ta xác định được giá trị tọa độ của các đối tượng đồ hoạ, từ đó chuyển đổi tọa độ của chúng sang hệ tọa độ mới. Để chuyển đổi bản đồ qua các múi chiếu có kinh tuyến trục khác nhau, căn cứ tọa độ $x, y$ theo công thức (3), (4) tính được tọa độ trắc đia $B, L$; theo công thức $(1),(2)$ chuyển toạ độ $B, L$ về tọa độ $x, y$ tương ứng với kinh tuyến trục mới. Khi chuyển đổi bản đồ số giữa các hệ tọa độ HN-72, VN2000, WGS-84, chúng ta sử dụng công thức (5), trong đó các hệ số chuyển đổi $A 1, B 1, C 1, \ldots A 2, B 2, C 2, \ldots$ được xác định dựa vào tọa độ các điểm song trùng trên cơ sở lý thuyết số bình phương nhỏ nhất [2].

Để chuyển đổi bản đồ từ hệ tọa độ HN-72 hoặc hệ tọa độ địa phương sang hệ tọa độ VN2000 đối với một khu vực không lớn, chúng ta xác định tọa độ cho 16 điểm ở cả 2 hệ tọa độ. Các tọa độ này được xác định trực tiếp hoặc thông qua phần mềm chuyển đổi tọa độ đã được cơ quan quản lý nhà nước công bố. Phần mềm tự động xác định được các hệ số biến đổi tọa độ theo công thức (5), từ đó chúng ta chuyển đổi tọa độ cho tất cả các điểm địa hình theo các hệ số vừa được xác định, giữ lại toàn bộ các thuộc tính của đối tượng địa hình.

Trong trường hợp khu vực chuyển đổi bản đồ có diện tích lớn, chúng ta thực hiện chuyển đổi theo từng mảnh bản đồ hoặc theo một đường địa vật rõ nét (đường, sông): (xem hinh 4). 


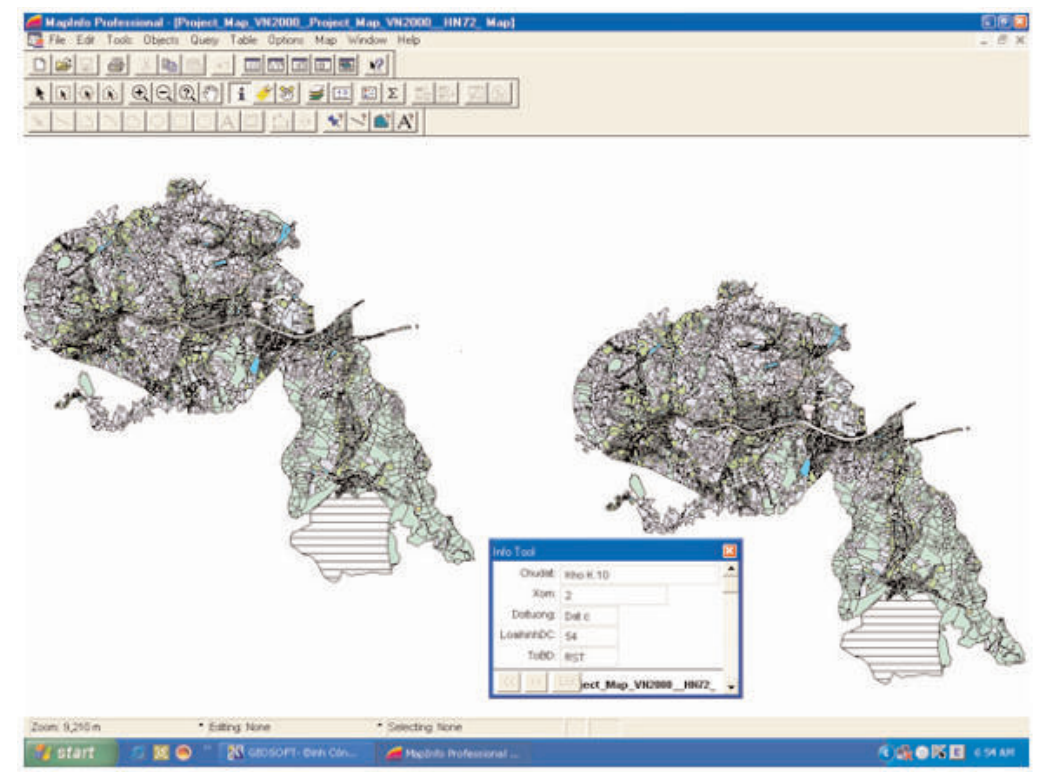

Hình 4: Kết quả chuyển đổi bản đồ từ hệ tọa độ HN-72 sang VN-2000

Trong trường hợp bản đồ được thành lập ở các kinh tuyến trục khác nhau, kích thước múi khác nhau, chúng ta tiến hành chuyển đổi tất cả các điểm địa hình từ tọa độ $X, Y$ sang tọa độ $B, L$ và cuối cùng chuyển về tọa độ $X, Y$ của múi chiếu mới trong cùng một hệ thống tọa độ HN-72 hoặc VN-2000. Trong đó dữ liệu được nhận từ phần mềm đồ hoạ AutoCad, MicroStation, Maplnfo.

\section{Kết luận}

Để thực hiện được những kết quả nêu trên, chúng tôi đã xây dựng các modul chương trình bằng ngổn ngữ lập trình Visual Basic 6.0, C++, các modul được kết nối với nhau tạo thành phần mềm ứng dụng tiện lợi và dễ sử dụng. Những kết quả nghiên cứu đã được ứng dụng trong thực tế sản xuất và kiểm tra tính chính xác thông qua các dữ liệu chuẩn. $O$

\section{Tài liệu tham khảo}

[1]. Đinh Công Hoà. 2011. Lập trình bài toán trắc địa cơ sở. NXB Giao thông Vận tải.

[2]. Nguyễn Trọng San, Đào Quang Hiếu, Đinh Công Hoà. 2002. Trắc địa cơ sở. NXB Xây dựng.O

\section{Summary}

\section{Applications of information technology for tunnel surveying, design, and construction}

Dr. Dinh Cong Hoa, MSc. Hoang Thi Thuy, University of Mining and Geology

Eng. Nguyen Van Lam, Tay Nguyen Minerals Mining Company, North Eastern Corporation, Vietnam Department of Defense

This paper focus on topographical data transformation between coordinate systems and automation in orientation determination and volume calculation for tunnel execution in traffic tunnel building, hydro-electric power plan, and mining based on geo-informatics. $O$

Ngày nhận bài: 17/10/2014. 